Revista Herencia, Vol. 33 (1), enero-junio, 2020.

\title{
LOCALIZACIÓN DE ÁRBOLES DE CHIRRACA (MYROXYLON PERUIFERUM, FABACEAE) EN SAN RAMÓN DE ALAJUELA A PARTIR DE LA NOVELA JUAN VARELA
}

\author{
Location of chirraca trees (Myroxylon peruiferum, Fabaceae) in San Ramón, Alajuela starting \\ from Juan Varela novel \\ Diego Aguilar-Sandí \\ Universidad de Costa Rica, Costa Rica \\ dd.10306@gmail.com
}

Recibido: $22-02-2020$

Aprobado: 27-03-2020

\begin{abstract}
Diego Aguilar Sandí realizó estudios en la Universidad de Costa Rica donde obtuvo el Bachillerato en Filosofía (2013) y el Bachillerato en Biología (2017). Actualmente es egresado de la Licenciatura en Biología con énfasis en Botánica, en la misma universidad.
\end{abstract}

\section{RESUMEN}

La presencia en Costa Rica de árboles de la especie Myroxylon peruiferum (Fabaceae), conocidos como chirracos o chirracas, se confirmó en el año 2019 con base en recolectas botánicas realizadas en los cantones de Acosta, Mora y Santa Ana. Hasta entonces, no había evidencia de su presencia en San Ramón de Alajuela. En noviembre de 2019 y enero de 2020, se hicieron dos recolectas de esta especie en la cuenca del río Barranca en San Ramón que fueron depositadas en el Herbario Luis A. Fournier Origgi (USJ) de la Universidad de Costa Rica. El objetivo de este artículo es evidenciar cómo a partir de la lectura de la novela Juan Varela, escrita por Adolfo Herrera García y publicada en 1939, se lograron ubicar esos árboles. Esta investigación estuvo orientada por la pregunta ¿puede una obra literaria servir de insumo para emprender una expedición botánica que permita ubicar una especie en un sitio determinado? Respuesta que resultó afirmativa en el caso que aquí se trata.

Palabras clave: árboles; exploración botánica; río Barranca; herbario USJ; historia natural; Myroxylon peruiferum; literatura; Juan Varela; novela

\section{ABSTRACT}

The presence in Costa Rica of Myroxylon peruiferum trees (Fabaceae), known as chirracos or chirracas, was confirmed in 2019 based on botanical collections made in the cantons of Acosta, Mora and Santa Ana. But, there was no evidence of their presence in San Ramón, Alajuela. In November 2019 and January 2020, two collections of this species were made in the Barranca river basin in San Ramón, which were deposited in the Luis A. Fournier Origgi Herbarium (USJ) of the University of Costa Rica. The objective of this article is to show how, from the reading of the novel Juan Varela, written by Adolfo Herrera García and published in 1939, these trees were located. This research was guided by the question: Can a literary work serve as input to undertake a botanical expedition that allows to locate a species in a certain place? Answer that was affirmative.

Keywords: trees; botanical exploration; Barranca river; USJ herbarium, natural history, Myroxylon peruiferum, literature, Juan Varela, novel 


\section{Introducción}

El conocimiento de la flora de un país inicia con la exploración botánica. Las recolectas de plantas que se realizan en estas expediciones forman parte de colecciones científicas que son custodiadas en herbarios y constituyen la fuente primaria de estudios florísticos y taxonómicos (Standley, 1937; Morales, 2012). Las muestras recolectadas pueden corresponder a especies ya descritas, pero nuevas para una región, o incluso a especies nuevas para la ciencia (Kriebel, 2005).

Una recolecta, debidamente herborizada, representa la evidencia de la presencia de un organismo de una especie determinada en un sitio específico. Por ejemplo, el hallazgo en Costa Rica de árboles de Myroxylon peruiferum, especie de la familia de las leguminosas (Fabaceae), está respaldado por ejemplares recolectados en Acosta, Mora y Santa Ana que se encuentran depositados en el Herbario Luis A. Fournier Origgi (USJ) de la Universidad de Costa Rica (Aguilar-Sandí, 2019). Esta especie fue descrita por Carlos Linneo el Joven en 1781, a partir de muestras recolectadas por José Celestino Mutis en Sudamérica ${ }^{1}$. Actualmente se sabe que su distribución natural abarca todo el neotrópico, desde el sur de México hasta el norte de Argentina (Sartori, Lewis, Mansano \& Tozzi, 2015). Sin embargo, no fue sino hasta el año 2019 cuando se confirmó su presencia en Costa Rica.

En Costa Rica, el nombre común de M. peruiferum es chirraco o chirraca (Aguilar-Sandí, 2019); nombre que asimismo reciben los árboles de $M$. balsamum, la otra especie del género, también conocida como bálsamo o sándalo (Fournier \& García, 1998; León \& Poveda, 1999). El término chirraca se utiliza únicamente en nuestro país y hace referencia a la resina que producen estos árboles² (Gagini, 1919; Diccionario Enciclopédico Quillet, 1974); de ahí que, un árbol de chirraca sea lo mismo que un chirraco.

\footnotetext{
${ }^{1}$ Linneo el Joven menciona, a propósito de la procedencia de las muestras recolectadas por Mutis, que esta especie "[h]abitat in calidissimis provinciis Terrae Firmae Americae meridionalis" (Linnaeus Filius, 1781, p. 233). Es decir, M. peruiferum "habita en regiones cálidas de tierra firme de América del Sur" (Traducción propia).

2 La resina de las dos especies de Myroxylon se ha utilizado en medicina tradicional y en la elaboración de productos comerciales (Revene, 2007). De estos árboles también se comercializa la madera, la cual es resistente a la pudrición y muy pesada (Lozenzi, 2002; Jiménez, Rojas, Rojas \& Rodríguez, 2011). En Costa Rica, el aprovechamiento de M. balsamum está vedado mediante el Decreto Ejecutivo N 25700-MINAE.
} 
La lectura de publicaciones científicas puede estimular el inicio de exploraciones; por ejemplo, la obra de Hans Weber, Los páramos de Costa Rica y su concatenación fitogeográfica con los Andes Sudamericanos (1959) representa, a juicio de Luis Diego Gómez, "el hito del cual arranca la exploración botánica sistemática de nuestros páramos" (2005, p. 104). A su vez, la obra de Paul C. Standley, Flora of Costa Rica (1937-1938), bien puede haber sido el aliciente que estimulara la visita de botánicos estadounidenses al país (Grayum, Hammel, Troyo \& Zamora, 2004). Pero, ¿puede una obra literaria servir de insumo para emprender una expedición botánica que permita ubicar una especie en un sitio determinado?

El objetivo de este artículo es evidenciar cómo a partir de la novela costarricense Juan Varela, escrita por Adolfo Herrera García y publicada en 1939, fue posible ubicar árboles de M. peruiferum en San Ramón de Alajuela; lo cual es una muestra de cómo las referencias que se encuentran en textos literarios de otras épocas permiten adquirir un conocimiento del entorno descrito por sus autores.

\section{Notas sobre la exploración botánica en San Ramón}

La historia de la exploración botánica en Costa Rica se remonta al siglo XIX con la llegada al país de Anders Oersted (Salazar, 2009). Sin embargo, el conocimiento sistemático de la flora costarricense iniciaría con la fundación del Museo Nacional de Costa Rica (1887) y con el arribo de Henri F. Pittier quien, entre otras acciones, promovió la fundación del Instituto Físico Geográfico Nacional (1889), cuna del primer herbario nacional (Gómez \& Savage, 1991).

Con el trabajo de Pittier y de sus colaboradores, entre los que destacaron Adolphe Tonduz, Paul Biolley y Carl Wercklé, las colecciones botánicas de este herbario llegarían a la cifra de "20 000 números, ca. del $60 \%$ de ellos acreditado a Tonduz" (Grayum, Hammel, Troyo \& Zamora, 2004, p. 12). Dicho material científico pasaría luego a formar parte del Herbario Nacional de Costa Rica adscrito al Museo Nacional (Gómez \& Savage, 1991).

Entre 1901 y 1902, Pittier contrató a Alberto Manuel Brenes encomendándole la preparación de "diez colecciones de plantas de quinientas especies cada una (...); trabajo que le significó recolectar alrededor de 5000 especímenes" (Salazar, 2009, p. 32); material que fue enviado al extranjero. Desde entonces, 
Brenes desarrollaría una importante carrera como recolector de plantas, aunque con un interés manifiesto por las orquídeas de San Ramón de Alajuela, su pueblo natal (Salazar, 2009). Sobre la labor de este ramonense, quien fue jefe de la Sección de Botánica del Museo Nacional entre 1920 y 1939, Paul C. Standley señaló que:

La región de San Ramón es ahora mejor conocida en herbarios que cualquier otra área de igual extensión en América Central (...). Qué afortunada sería la ciencia botánica si tan solo hubiese más recolectores con igual ahínco y discernimiento. (Traducción propia) ${ }^{3}$.

¿Habrá Alberto Manuel Brenes recolectado muestras de árboles de chirraca en San Ramón? Si lo hizo o no lo hizo, nunca lo sabremos. La recolecta más antigua que se conserva del género Myroxylon en Costa Rica procede de San Mateo de Alajuela y fue realizada por Otón Jiménez en 1918. Esta muestra, resguardada en el Herbario Nacional de Costa Rica, corresponde a un ejemplar de $M$. peruiferum, aunque su identidad taxonómica no se estableció sino hasta un siglo después de haber sido recolectada (Aguilar-Sandí, 2019).

"Entre 1940 y 1968 existió un vacío en la investigación biológica en la región del cantón de San Ramón" (Gómez-Laurito \& Ortiz, 2004, p. 115), que vendría a subsanarse con la creación de la Reserva Forestal de San Ramón en 1975, sitio que desde entonces se convirtió en el destino predilecto de los investigadores que realizaron exploraciones botánicas en este cantón alajuelense. Dicha área protegida, ubicada en el distrito de Los Ángeles, se cambió a la categoría de reserva biológica en 1993, pasando a llamarse Reserva Biológica Alberto Manuel Brenes (ReBAMB) (Salazar, 2009).

La microcuenca de los ríos San Lorenzo y San Lorencito, extensión que representa un $5 \%$ del área total de la ReBAMB, ha sido el sector más explorado en esta área protegida. En el trabajo de Gómez-Laurito \& Ortiz (2004) se reportaron 1294 especies de plantas de 483 géneros y de 137 familias en esa zona. Sin embargo, pese a que este estudio incluyó información recopilada durante 26 años (1978-2004), no se reportó $M$. peruiferum; lo que indica que esta especie no fue encontrada allí.

3 "[T]he San Ramón region is now better known in herbaria than any other area of equal extent in Middle America (...). How fortunate botanical science would be if only there were more collectors of equal industry and discrimination" (Standley, 1937, p. 53). 


\section{El río Barranca y los árboles de chirraca en Juan Varela}

El protagonista de la novela, Juan Varela Conejo, es un jornalero oriundo de Santa Bárbara de Heredia que denuncia un terreno "situado en las bajuras de la Barranca, distrito segundo del cantón segundo de la provincia de Alajuela" (Herrera García, 1983, p. 15). Es decir, en Santiago de San Ramón. Juan y su novia Ana emigran hasta allí para iniciar una vida conjunta. Hay constancia de que el autor conoció esta zona, pues trabajó como administrador de una mina de oro ubicada en las montañas de Santiago ${ }^{4}$.

Al llegar al sitio indicado, Juan inicia el desmonte del terreno con el objetivo de establecer una finca, de la que él y su familia obtendrán el sustento:

\footnotetext{
Y toda la semana con las manos agarrotadas a la herramienta volteando montaña en medio de un silencio que se rajaba como los troncos a hachazos. Apenas había tiempo de sembrar el maíz y regar los frijoles. Ana le ayudaba: inclinada sobre el campo iba desparramando puñados de granos, mientras él, más adelante, abría tierra de sembradura entre la montaña tupida. (Herrera García, 1983, p. 19)
}

Si tomamos en cuenta que a principios del siglo XIX "se inició la colonización agrícola primaria de San Ramón" (Meza \& Bonilla, 1990, p. 60), entonces cabe suponer que el personaje de Juan Varela está inspirado en uno de los colonos de esta región, a juzgar por el hecho de que Juan debe voltear montaña antes de establecer los campos de siembra ${ }^{5}$. Sin embargo, sería un colono que llega de forma tardía, pues el denuncio se lleva a cabo a inicios del siglo $X^{6}{ }^{6}$. La propiedad adquirida es, en efecto, una de las más recónditas, lo que da la impresión de que las mejores localidades ya estaban ocupadas: "¿La bajura de la Barranca? Mala tierra. Mucha culebra. Agarren por allí, por aquel camino que se pierde al sur. Cuatro horas a pie." (Herrera García, 1983, p. 16).

\footnotetext{
${ }^{4}$ En la 3era y 4ta edición de Juan Varela se incluye una nota biográfica sobre Herrera García, donde se señala que "Después de seis años de trabajar en el Diario La Hora, decide abandonar el periodismo y en 1939 lo cambia por el de Capitán de Mina (una especie de administrador) en una mina propiedad de sus tíos don Gonzalo Moncada y de doña Armandina García Solano. La mina de oro estaba ubicada en los Bajos de San Gerardo, al suroeste del cantón de San Ramón en Alajuela" (Anónimo, 2009, p. 82). Por otra parte, en Piedades Sur (distrito quinto) existe un sitio llamado Barranca y otro llamado Bajo Barranca, quizá el autor se esté refiriendo a alguno de estos lugares, próximos a lo que hoy es Santiago.

${ }^{5}$ La literarura es ficción, no obstante, aquí se asume que la referencialidad que en ella se articula responde a aspectos propios del contexto en que es producida.

${ }^{6}$ En la 1era y en la 2da edición de la novela no se indica el año exacto del denuncio, tan solo se menciona que fue suscrito el "29 de enero de 19..." (sic.) (Herrera García, 1983, p. 15). En la 3era edición se agrega el año de "1935". Sin embargo, en la 2da reimpresión de la 3era edición, así como en la 4ta edición, se mantiene el texto de la 1era edición.
} 
Las abras, volteas o desmontes son parte de la historia de la colonización campesina del Valle Central de Costa Rica. En general, de lo que una vez fueron bosques prístinos, hoy solo quedan parches boscosos inmersos en un ambiente urbano-rural (Heuveldop \& Espinoza, 1983; Cascante-Marín \& Estrada-Chavarría, 2012).

Los estudios literarios sobre Juan Varela coinciden en que la obra tiene un carácter de denuncia social, pues en ella se expone el tema del despojo y de la explotación financiera que padeció el campesinado costarricense (González, 2010; Cubillo, 2016). Sin embargo, la novela también permite abordar temas de historia natural, ya que, además de las vicisitudes de Juan, que van en detrimento de su persona, de su familia y de su patrimonio7, Herrera García introduce en la narración elementos geográficos, zoológicos y botánicos.

La travesía de Juan y Ana, desde Santa Bárbara de Heredia hasta Santiago de San Ramón, es un viaje hacia la vertiente Pacífica de Costa Rica; este aspecto se ilustra con la referencia al cambio en el relieve y al avistamiento del golfo de Nicoya desde las montañas santiagueñas.

El camino que se perdía al sur era un repecho infinito. La tierra, agobiada de lucir airosa en la Meseta Central, se echaba de bruces, desgreñada de cascajos, para mojarse en el Pacífico. (...) Sobre un altozano, a dos horas de San Ramón, descansaron. Muy remoto, muy brillante, muy quieto, el Golfo de Nicoya. (...) Antes de amanecer se oía la correntada del río. Estaban en su tierra. (Herrera García, 1983, p. 16)

Yo mismo atestigüé cómo, desde el pueblo de Magallanes en Santiago y desde las montañas de Piedades Sur de San Ramón, es posible observar a simple vista el golfo de Nicoya y la sección sur de la península de Nicoya (Fig. 1, A). Comprobación que le da un acento naturalista al relato, junto con las referencias al río Barranca y a la montaña San Gerardo; sitio donde operó la mina San Gerardo, mejor conocida en la actualidad como mina Moncada (N. Chaves, com. pers., 2020), misma de la que fue administrador don Adolfo.

Allí estaba la vida, toda la vida: el río Barranca, entre torrentadas pétreas, al pie meridional de la finca, cantaba su canción de cuarzo. Nace una hora más al norte. Recoge en su lecho angosto y profundo las aguas cantoras de la montaña de San Gerardo y al bajar a la llanada de Esparta aquiétase en un estirón plácido y ampuloso que se ensancha más aún hasta caer en los playones del océano. (Herrera García, 1983, p. 26)

\footnotetext{
${ }^{7}$ La vida de Juan Varela está marcada por una serie de desgracias. Grosso modo, pierde su finca, con lo cual pasa de ser propietario a ser peón. Luego, tras perder su trabajo de peón, instala una saca de guaro, con lo cual queda a merced de la ilegalidad de este oficio; en defensa del cual llegará a asesinar a un agente del Resguardo Fiscal, crimen que penará de por vida en la cárcel de San Lucas.
} 
A partir de estas referencias geográficas, puede inferirse que las montañas de la cuenca del río Barranca son el escenario donde se ambienta la historia de Juan Varela (Fig. 1, B). Y por lo tanto, son el sitio donde posiblemente se encuentren los árboles de chirraca. Herrera García menciona estos árboles en dos ocasiones: la primera cuando Juan Varela levanta el rancho en el abra, y la segunda cuando los jornaleros llegan a beber guaro a la saca de Juan.

Hacia el centro de la selva la tierra se acurrucaba píamente. Allí clavó cuatro horcones -cedro, chirraca, níspero y treshuevos-, los entechó de paja y encendió el fogón. ¡Su casa! (Herrera García, 1983, p. 19)

En grupos paralelos han ido bajando los hombres a la Barranca (...). En el propio bajo, en un recoveco de rocas, se oye una batahola de gritos y carcajadas. De vez en vez, un güipipía desafiante asciende hasta las copas de los chirracas y cae al ribazo de enfrente, repitiéndose su retumbo de barranco en barranco. (...) Un trillo que se retuerce desesperadamente entre las rocas macizas y los charralones espinosos, en el corazón mismo de San Gerardo Viejo, remata en la saca de Juan Varela. (Herrera García, 1983, pp. 53-54) ${ }^{8}$.

Como puede apreciarse, el espacio narrado en Juan Varela es un espacio ubicable. Aspecto que permite a un explorador contemporáneo localizarlo y recorrerlo. En casos como este, no debe perderse de vista que las obras literarias son ficción, aunque tengan como telón de fondo un contexto histórico - naturalista. "La narrativa literaria está organizada según un principio dramático y su verosimilitud depende exclusivamente de la capacidad del escritor para convencer a los lectores" (Molina, 2014, p. 4). Reconozco que Herrera García me convenció. De ahí que, luego de leer la novela, me planteara dos preguntas: ¿Es posible encontrar árboles de chirraca en las montañas ubicadas en la cuenca del río Barranca en San Ramón de Alajuela? En caso afirmativo, ¿corresponderán a la especie M. peruiferum?

\footnotetext{
${ }^{8}$ El destacado en negrita no está en el original.
} 
Figura 1. Paisajes mencionados en la novela Juan Varela. A. Vista del golfo de Nicoya y de la sección sur de la península de Nicoya desde las montañas de San Francisco de Piedades Sur, San Ramón, Alajuela. B. Montañas de Santiago de San Ramón en la cuenca del río Barranca.

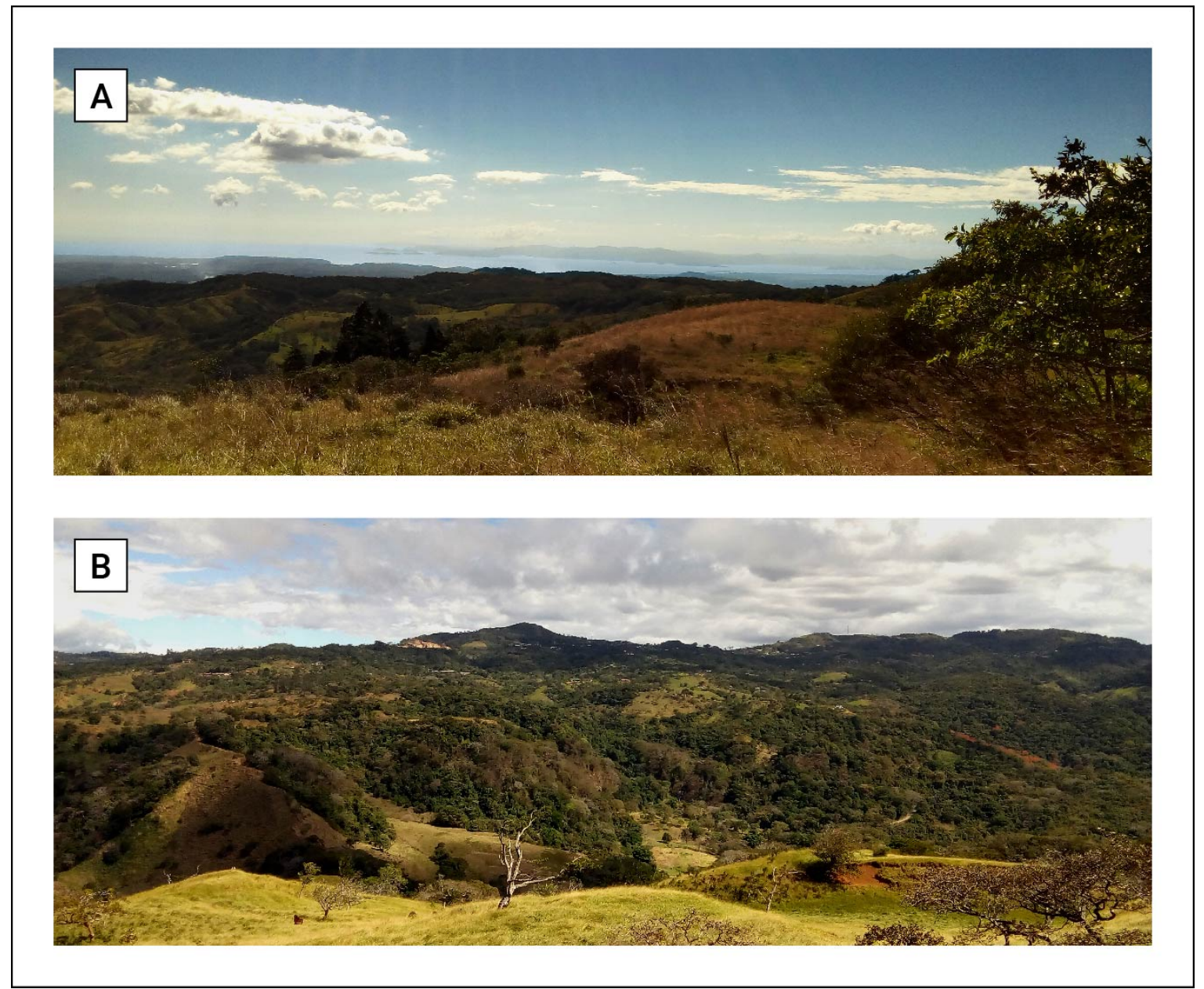

Fuente: Diego Aguilar Sandí.

\section{Hallazgo de árboles de chirraca en San Ramón de Alajuela}

El 23 de noviembre de 2019 y el 18 de enero de 2020 visité San Ramón en compañía de los biólogos Nelson Chaves y Alejandro Quesada. Trabajamos bajo el supuesto de que si las referencias de Juan Varela tenían un asidero naturalista, entonces los árboles de chirraca formarían parte del paisaje de las montañas cercanas al río Barraca.

La metodología consistió en dirigirnos a las montañas de Santiago e identificar los árboles que encontráramos a nuestro paso. Además de preguntar a las 
personas que vivían en esos lugares si conocían dichos árboles o si en algún momento de sus vidas habían escuchado sobre ellos; con lo que obtendríamos señas más específicas sobre su ubicación.

El 23 de noviembre recorrimos el poblado de Río Jesús de Santiago. Ahí nos informaron que don Francisco Rodríguez podía ayudarnos debido a su conocimiento sobre los árboles de la zona. Logramos contactarlo. Él nos aseguró que había visto chirracas en las faldas de las montañas de Piedades Sur. Además, nos facilitó el contacto de Carlos Espinoza, quien conocía un sitio específico donde había chirracas en el distrito de San Rafael: allí encontramos, efectivamente, un árbol de la especie $M$. peruiferum que había sido cultivado hacía aproximadamente 30 años, pero Espinoza nos aseguró que las semillas procedían de árboles de la zona.

Para el 18 de enero, Alejandro Quesada había contactado a Diego Zamora y a don Francisco Zamora, quienes habían visto chirracas en San Francisco de Piedades Sur. Expresamente nos dirigimos a conversar con ellos. Francisco Zamora había sembrado una chirraca hacía tres años y conocía el sitio exacto donde se encontraba el árbol del que había recolectado las semillas. Nos condujo hasta él. ¡Era un árbol de $M$. peruiferum de aproximadamente $30 \mathrm{~m}$ de altura y $60 \mathrm{~cm}$ de DAP'!

En los dos días de gira, logramos ubicar tres árboles de M. peruiferum (Fig. 2). De dos de ellos hice recolectas botánicas que deposité en el Herbario Luis A. Fournier Origgi (USJ). Como es requisito en la preparación de ejemplares de herbario, cada muestra está acompañada de una etiqueta en la que se consigna la localidad, la fecha, el nombre del recolector, el número de recolecta y cualquier otra característica de la planta in situ que no sea visible en la muestra o que pueda perderse tras el secado (Rodríguez, 1969).

Información de los ejemplares de herbario: Costa Rica. Alajuela, cantón San Ramón, distrito San Rafael, Rincón de Mora, 1002'41.6' N, 84²8'22.4' 'W, 1200 msnm, 23 noviembre 2019, Aguilar-Sandí 16 (USJ); distrito Piedades Sur, San Francisco, 1004'40.3"N, 84³2'27.6"W, 900 msnm, 18 enero 2020, AguilarSandí 18 (USJ).

\footnotetext{
${ }^{9}$ El diámetro a la altura del pecho (DAP) es una medida utilizada para cuantificar el grosor de los troncos de los árboles. Consiste en medir la circunferencia del tronco a la altura del pecho de una persona (aproximadamente a 1,3 m del suelo) y dividir ese valor entre $\pi$.
} 
Figura 2. Árboles de chirraca (Myroxylon peruiferum, Fabaceae) encontrados en San Ramón de Alajuela. A. San Francisco, Piedades Sur (Ejemplar Aguilar-Sandí 18, USJ). B. San Francisco, Piedades Sur (Fotografía de A. Quesada, en la que aparece el autor junto a un árbol de aprox. $30 \mathrm{~m}$ de altura y $60 \mathrm{~cm}$ de DAP). C. Rincón de Mora, San Rafael (Ejemplar Aguilar-Sandí 16, USJ).

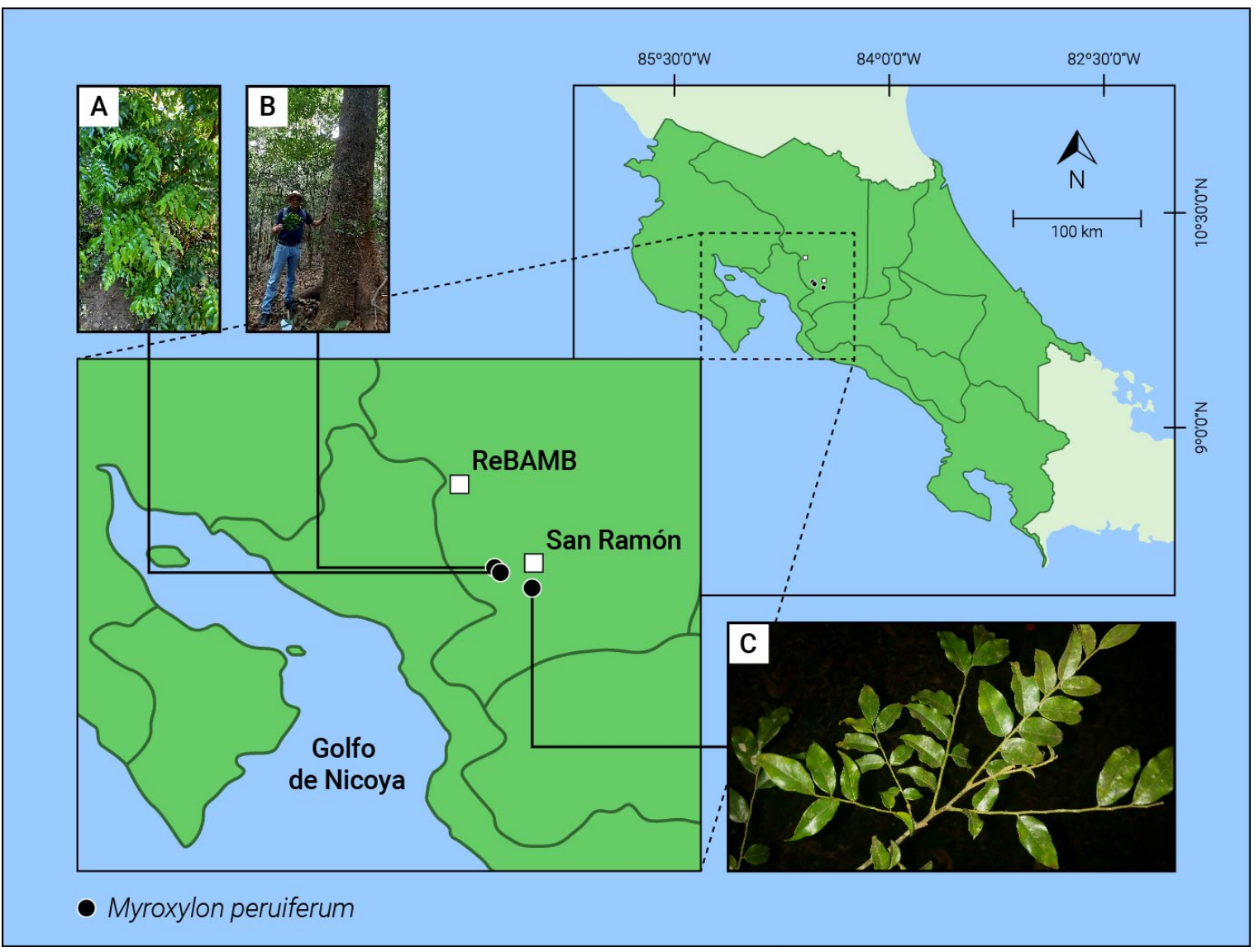

Fuente: Diego Aguilar Sandí.

\section{Consideraciones finales}

La respuesta a la pregunta que motivó la redacción de este artículo fue afirmativa: la riqueza de la herencia literaria de Juan Varela sirvió como insumo para emprender una expedición botánica que permitió ubicar árboles de $M$. peruiferum en la cuenca del río Barranca. Este hallazgo permite plantear nuevas investigaciones, ya que se desconoce el tamaño y la diversidad genética de las poblaciones de esta especie en esa región (en particular) y en el país (en general). 
En ese sentido, el recurso literario puede servir para emprender investigaciones similares con otras novelas como El sitio de las abras de Fabián Dobles (1950), Murámonos, Federico de Joaquín Gutiérrez (1973) y La loca de Gandoca de Anacristina Rossi (1992), en las que se hace mención de nombres comunes de árboles y de sitios ubicables geográficamente en Costa Rica, una posibilidad de rescatar y transformar el patrimonio literario en instrumento para la botánica.

\section{Agradecimientos}

Aunque Juan Varela me llevó a la cuenca del río Barranca, lo cierto es que sin la ayuda de Francisco Rodríguez, Carlos Espinoza, Diego Zamora y Francisco Zamora no hubiese llegado al pie de cada árbol de chirraca. Este hallazgo tampoco habría sido posible sin la ayuda de Nelson Chaves y Alejandro Quesada, quienes conocen San Ramón como a la palma de sus manos. Agradezco también a Elmer G. García por haber revisado el borrador de este artículo. Por último, quiero agradecer muy especialmente a Cecilia Elizondo y a Javier Chaves por haberme hospedado en su hogar. Fueron días espléndidos.

\section{BIBLIOGRAFÍA}

Aguilar-Sandí, D. (2019). El árbol Myroxylon peruiferum (Fabaceae), nuevo registro en la flora de Costa Rica. UNED Research Journal, 11(3), 424-429.

Anónimo. (2009). Sobre el autor y sus obras. Herrera, A. Juan Varela ( $4^{\circ}$ ed., pp. 81-87). San José, Costa Rica: Editorial Costa Rica.

Cascante-Marín, A. \& Estrada-Chavarría, A. (2012). Las plantas vasculares de El Rodeo, Costa Rica. Brenesia, 77, 71-128.

Cubillo, R. (2016). Representaciones de la pobreza y la desigualdad social en la narrativa costarricense de la Generación del 40. Memorias: Revista Digital de Arqueología e Historia desde el Caribe, 12(30), 10-38.

Diccionario Enciclopédico Quillet. Tomo 3. (1974). México: Editorial Argentina Aristides Quillet. 
Fournier, L. A., \& García, E. G. (1998). Nombres vernáculos y científicos de árboles de Costa Rica. San José, Costa Rica: Editorial Guayacán.

Gagini, C. (1919). Diccionario de costarriqueñismos (2a ed.). San José, Costa Rica: Imprenta Nacional.

Gómez, L. D., \& Savage. J. M. (1991). Investigadores en aquella rica costa: biología de campo costarricense 1400-1980. D. H. Janzen (ed.). Historia Natural de Costa Rica (pp. 1-11). San José, Costa Rica: Editorial de la Universidad de Costa Rica.

Gómez-Laurito, J., \& Ortiz, R. (2004). Lista con anotaciones de las Angiospermas de la Reserva Biológica Alberto Brenes (Microcuencas de los ríos San Lorenzo y San Lorencito), Costa Rica. Lankesteriana, 4(2), 113-142.

Gómez, L. D. (2005). La exploración científica de los páramos costarricenses. En M. Kappelle, \& S. P. Horn (eds.). Páramos de Costa Rica (pp. 101-110). Santo Domingo de Heredia, Costa Rica: Instituto Nacional de Biodiversidad.

González, I. (2010). Juan Varela, de Adolfo Herrera García: un alegato a favor del Homo oeconomicus. Filología y Lingüística, 36(1), 105-118.

Grayum, M. H., Hammel, B. E., Troyo, S., \& Zamora, N. (2004). Historia/History. En B. E. Hammel, M. H. Grayum, C. Herrera, \& N. Zamora (Eds.), Manual de Plantas de Costa Rica. Vol. I. Introducción (pp. 1-50). Monographs in Systematic Botany from the Missouri Botanical Garden, 97, 1-299.

Herrera, A. (1983). Juan Varela ( $1^{\mathrm{a}}$ ed., $11^{\mathrm{a}}$ reed.). San José, Costa Rica: Editorial Costa Rica.

Heuveldop, J., \& Espinoza, L. (1983). El componente arbóreo en Acosta y Puriscal, Costa Rica. San José, Costa Rica: Centro Agronómico Tropical de Investigación y Enseñanza (CATIE).

Jiménez, Q., Rojas, F. E., Rojas, V., \& Rodríguez, L. (2011). Árboles maderables de Costa Rica. Ecología y silvicultura ( $2^{a}$ ed.). Heredia, Costa Rica: Instituto Nacional de Biodiversidad. 
Kriebel, R. (2005). Una nueva especie y un nuevo registro de Drymonia (Gesneriaceae) en Costa Rica. Lankesteriana, 5(1), 81-83.

León, J., \& Poveda, L. J. (1999). Los nombres comunes de las plantas en Costa Rica. Heredia, Costa Rica: EUNA.

Linnaeus Filius, C. (1781). Supplementum Plantarum. Brunsvigae: Impensis Orphanotrophei.

Lorenzi, H. (2002). Brazilian trees. A guide to the identification and cultivation of brazilian native trees. Vol. 1 ( $4^{a}$ ed.). São Pablo, Brasil: Instituto Plantarum.

Meza, T. A., \& Bonilla, A. (1990). Áreas naturales protegidas de Costa Rica. Cartago, Costa Rica: Editorial Tecnológica de Costa Rica.

Molina, I. (2014). Narración histórica y narrativa literaria. A. P. Malavassi (comp.), Historia: ¿ciencia, disciplina social o práctica literaria? (pp. 3-11). San José, Costa Rica: Editorial de la Universidad de Costa Rica.

Morales, C. O. (2012). El Herbario USJ de Costa Rica: trayectoria y contribuciones. Revista de Biología Tropical, 60(4), 1641-1648.

Revene, Z. (2007). Los Balsameros de Atiluya, Sonsonate, El Salvador. El Salvador Investiga, 3, 5-23.

Rodríguez, R. L. (1969). Apuntes y guía de laboratorio de botánica sistemática. Serie de textos $N^{\circ} 179$ ( $3^{a}$ ed.). San José, Costa Rica: Publicaciones de la Universidad de Costa Rica.

Salazar, A. H. (2009). Alberto Manuel Brenes, el naturalista. San José, Costa Rica: Editorial de la Universidad de Costa Rica.

Sartori, A. L. B, Lewis, G. P., Mansano, V. F., \& Tozzi, A. M. G. A. (2015). A revision of the genus Myroxylon (Leguminoseae: Papilionoideae). Kew Bulletin, 70, 48. doi: 10.1007/S12225-015-9604-7

Standley, P. C. (1937). Flora of Costa Rica. Botanical Series, vol. 18. Chicago, USA: Publication of Field Museum of Natural History. 
Revista Herencia, Vol. 33 (1), enero-junio, 2020. 